\title{
Tropical Nusantara's Contemporary House for Liveable Environment
}

\author{
Agung Murti Nugroho, ${ }^{1, *}$ \\ ${ }^{1}$ Department of Architecture, Engineering Faculty, 65145 University of Brawijaya, Indonesia
}

\begin{abstract}
The tropical house has been discussed in many journals in various architectural perspective. Tropical Nusantara's Contemporary House is an alternative style in Indonesia as the livable environment in the present. This paper seeks to raise more specific points about tropical Nusantara's house with topics covered, such as identity style of our house; tropical Nusantara's house characters; tropical Nusantara's house design creations as well as cultural knowledge in habitat. Hopefully, this paper can provide an alternative thinking about the tropical Nusantara's house, not only as a habitable space but also can provide a comfortable dwelling and give satisfaction to settlement.
\end{abstract}

\section{Nusantara's contemporary house as identity}

The style of house in the present days in Indonesia has various models and types of design that is diverse, such as classic, modern, and contemporary. Selection of house design style considers compatibility with climate, occupant comfort needs, and identity of the occupant. Non-conformity with the tropical climate will have an impact on the expensive cost of building operations and treatment. The non-fulfillment of occupant comfort needs has an impact on the addition of an artificial conditioning system which consequently is a high cost of building operational cost. While the identity aspect is associated with the pride and satisfaction on the characteristics of the house that differentiates with other houses. In general, seen from the development of the house design style in Indonesia, it follows three periods of the architectural style that is before colonization, colonial period, and after independence or can be classified in the style of traditional houses. The traditional house style is very much divided into vernacular style and traditional classic style. The vernacular style is an architectural style that is naturally formed by the community and there are many in traditional villages spread throughout the Nusantara archipelago. Meanwhile, the traditional classic style has rules and traditions that are clear and spread through generations, either through certain rituals or written in ancient records. The variety of vernacular and traditional styles when viewed more specifically, then there are more than 500 types of traditional houses with an assumption that this number is half the number of ethnic groups in Indonesia for 1,128 groups (source of BPS). In the present era, the number of traditional house types tends to diminish both that has been destroyed and moved by owners and location. The style of house that stands out in the Colonial era is the Hindis

* Corresponding author: sasimurti@yahoo.co.id 
house. Hindis house style features high ceiling spaces, wide enough eaves, and the presence of verandas both in front and behind the building as a form of adaptation to local conditions and cultures. Although Colonial house had a stylistic development, it had an impact on its uniformity in the form of a single house with a precarious roof, a stucco wall on a layer of stone trash, a window of teak wood, and there is also a two-story building with a garage and a pavilion. This is different than Traditional houses that are very diverse and able to express the identity of each culture. The house style after Indonesian independence, especially after 1960, is quite diverse among others, which stands out as a Jengki style. The Jengki style is characterized by the use of a saddle roof that is peaked, the dominant view of the line and the incline, as well as the use of local materials. In the era of 90's, Mediterranean style came that is actually a style of architecture originated in Spain (which is the Mediterranean region, Southern Spain), it is popular in the United States and extends throughout the world.

Characteristic of Mediterranean buildings are appropriate for hot climates with thick walled buildings to protect from the heat during the day, but stay warm during the night; small windows to withstand the heat' and the arch elements of windows and doors as a continuation of Spanish style that once hit Indonesia before. While the last style of the house that very much preferred is Minimalist style. Viewed from the historical aspect, this style was born in the 18th century and flourished in Indonesia in the 2000 s by no longer following the original grip, but experienced adjustments to local conditions. So, this style is the result of combination with other elements into a minimalist tropical style, minimalism ethnic, contemporary minimalism, and so forth. The essence of Minimalist style is the simplicity of the form with a little composition of the mass. Aesthetics are generated through harmonious small comparisons, a combination of monotonous textures in geometric forms. Spatial inside is arranged with the placement of furniture that does not meet the space and there is continuity between spaces without space divider to produce a broad impression and fused. The windows and doors size are large and simple.

Based on many house styles and their development, they come into the fundamental questions, they are: First, how long the style can survive the climate so it is able to minimize building maintenance. Second, how far can comfort the occupants without the help of artificial systems? Third, how the style can be an identity or give pride/satisfaction to the occupant. The tropical climate as a natural feature of life that can not be denied must be an early consideration in building a house. In the making of the house should also able to give comfort to its occupants. Each of the house occupants has a different history, origin, tribe, culture, and identity. The comfort of tropical Nusantara's house has a high comfort tolerance, it means it has a large adaptation rate, especially to high temperature and humidity conditions. Based on some studies, this tolerance number is smaller because many of us are used to live in an air-conditioned environment. Lifestyle in big cities makes most of the time we are in an air-conditioned environment, starting from a living house, in vehicles, at work all use the air conditioner. So, the demands of our comfort numbers change to be higher. As a result, our adaptation rates are lower and more vulnerable to climate change, such as illness and lower work productivity.

Tropical Nusantara's nuance as an alternative to present style, not only provides a variety of appearance / building style, but also a variety of styles and cultures of life that climate responsive, energy efficient, and conscious culture that became an alternative answer to various problems, climate change, energy crisis, and identity crisis. Judging from the historical development of architecture as having been parsed in front, this Tropical style has emerged several times. As in traditional times, almost most of the Traditional house styles have adapted to the tropical climate. Similarly, the Hindies style in which the Dutch style buildings have been tried to adapt to tropical conditions. In the Modern style, several generations of architects have tried with attention to tropical climate aspects. The idea of 
Nusantara has long been buzzing not only in the past but also encompassing the present and the future. Nusantara as a container of Indonesia nature with the basis of the personality of the perpetrators who follow the noble values of the Nation, it would naturally form a built environment characteristic of Indonesia. So, with the unity of the word "Tropical" and "Nusantara" is integral as the focus of making the form, space, and place that gives knowledge of a new culture. Tropical Nusantara's house continues the typical Indonesian inhabited tradition that reflects the lifestyle, the meaning of life and culture of life. Tropical Nusantara is not just an alternative to the new present style but also gives the spirit to continue the tradition of the past. This style not only repeats the form but also contains the values and noble knowledge, so it becomes a real identity both individual and social. The identity of Indonesian houses is not simply seen from the look of the style, but how the house that is created able to create a comfortable lifestyle and become a living culture that provides the means of learning for the family as the smallest group of people, to transfer knowledge of noble values of the Nation.

\section{Tropical Nusantara's house: more than a living space}

As mentioned earlier, Tropical Nusantara's house is not just a family room, but also a comfortable place to live and give life happiness. This comfort is closely related to the climatic conditions in Indonesia, which is characterized by humid tropical islands. So that the tropical conditions of Nusantara provide unity in the housing diversity of the past, began linguistically to the shape of the building. Linguistically, there is a vocabulary in Tropical Nusantara that has the same origin, for example, the word "house", in Java called "omah", in Nusa Tenggara called "uma", in Minangkabau called "rumah". Similarly, the influence in the concept and form of tropical houses, for the Nusantara's people, the house is not just a place to live, but it is a building that shows a number of important ideas of the embodiment of knowledge in the past. So, it is a fitting house as the first place to learn about the noble values of the Nation.

In the beginning, as a form of living tradition, the house is only limited to a shelter that gives the living space of its occupants. Subsequent developments are increasing into a comforting residence. As the family grows, the house becomes a source of knowledge. House is seen as a form of culture based on human thinking and extends to families, communities, society. As in the first series on the definition of Nusantara, then Nusantara's house formed by the conditions of occupants and governance in an archipelago tropical natural environment, empirically or fact. The tropical climate in Nusantara has characteristics of high rainfall, great solar radiation, temperature differences, and less air humidity in the day and night. Tropical Nusantara has a specific character due to its position on the equator, between two continents and two oceans, and is an archipelagic environment that has high humidity and temperature compared to other tropical regions. Tradition of Tropical Nusantara's house has characteristics: 1) accuracy in choosing the position of the house on the site; 2) harmony of building skin in response to climatic conditions through the use of porous elements or better known as "breathing"; 3) the integration of shelter space; 4) the unity of place and the utilization of natural materials as time changes; 5) the simplicity of knowledge in adapting to the environment. The appropriate foothold in determining the position of the house as an example is the appropriate selection of treads and soil levels, consideration of wind direction, and the slim shape of the age of the building associated with the circulation of the sun. The harmony of the building's breath lies in the form of the roofs, walls, and floors that use hollow materials. The alignment of shaded space through the wide roof shape, the oblique wall, and the height variation of the stage. The togetherness of place is a commonplace through the arrangement of the distance between houses. The simplicity of knowledge is the 
culmination of a past tradition that should be sustainable. The tradition of Tropical Nusantara's house not only covers the microscope inside the house or microscope outside the house but also the wider environment in the settlement area. Tropical Nusantara's house ultimately gives a picture of specific space atmosphere that gives the impression and happiness of living as a result of the utilization of the site potentials., the occupants comfort and the shared awareness to improve the environment. The terminology of Tropical Nusantara House can be explained in Figure 1, which is through climate-responsive dwellings, comfortable living spaces, and pleasant habitable culture.

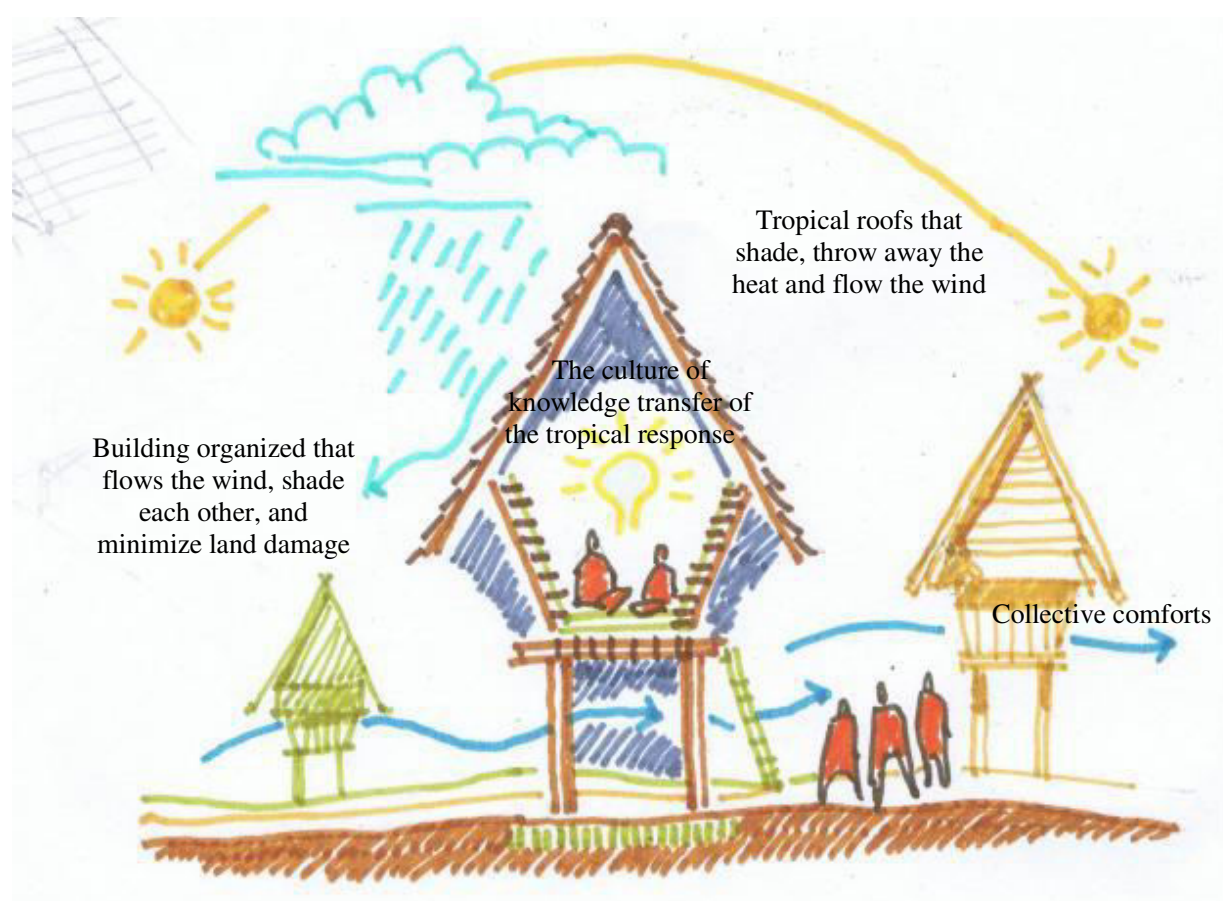

Fig. 1. The tradition of Tropical Nusantara's house as a climate responsive living space, comfortable living space, and a pleasant living culture.

\section{Tropical Nusantara's house characters}

This study explains climatic characters that are evident in the architecture of the tropical house characters in the South East Asian archipelago region. The aim of this study is to give information about the tropical Nusantara's architecture and to investigate the relationship between the architectural products and the climate that plays a very important role in the formation of this architecture. Thus, the effects of climatic factors, such as solar radiation, temperature, humidity, wind, and rainfall, on Nusantara's house are explained in the shape, shade, material.

\subsection{Tropical climate characters}

Tropical Nusantara's house character cannot be separated with the aspect of tropical climate character and the tropical house itself. Tropical climate character consists of four main interrelated components: solar radiation, temperature and humidity, wind, and rainfall. 
These characters mainly affect the house construction in the tropics ranging from aspects of planning, development, maintenance, and demolition. The four components are a unity of the natural system, solar thermal radiation will cause changes in temperature and humidity so that the difference in temperature and air pressure will cause air movement or called wind. Changes in solar radiation, temperature, humidity, and wind will affect rain patterns and so forth.

Solar radiation intensity varies in every place that is strongly influenced by the angle of the illuminated field and the radiation distribution of a building element. So, the consequence of avoiding high solar radiation in buildings is the presence of direct sunlight protection elements. The main factors that affect solar radiation are the sun intensity, time and movement of the sun. Sun intensity is highly dependent on the wavelength of radiation, the surface area and the level of solar heat. The sun position is manifestly produces based on altitude and azimuth angle. The altitude is the angle between the horizontal surface and the direct sunlight. Meanwhile, the azimuth gives the position of the sun measured from the North to the East or West. The height of the sun varies with latitude, because the earth is spherical, so the angle falls in different rays over time at that location. The sun movement is mainly determined the sphere of the orbit of the sun on earth and the angle between the equator and the solar orbit. Between March 21 and September 24, the sun spins over the equator. These days, it is known as equinox days, so the area along the equator takes maximum intensity on a normal surface against the direction of radiation. On June 22, the sun positions us at the Northern solstice and on December 21, the sun position is at the Southern turning point. During the period of a turning point, the sun is at the furthest from the equator.

Temperature is a measure of the degree of heat intensity. The temperature difference between two points provides the potential for allowing heat transfer from warmer points to cooler points. In equatorial regions, the annual temperature range is very small and ranges from $2^{\circ} \mathrm{C}$ to $3^{\circ} \mathrm{C}$. Meanwhile, the daily temperature has a larger range of $5^{\circ} \mathrm{C}$ to $8^{\circ} \mathrm{C}$ in the coastal area and $8^{\circ} \mathrm{C}$ to $12^{\circ} \mathrm{C}$ in land area. While air humidity is the amount of water vapor in a given space. High humidity can slow evaporation through sweat and breathing. The drier and hotter the air, the faster the flow rate of heat release from the skin to the air. The comfort limit for humidity is $40 \%$ to $60 \%$. In daily life cycle, the relative humidity is the opposite of temperature, i.e. maximum humidity occurs in the morning and minimum humidity occurs in the afternoon.

The wind has an effect on the heat release on the body, either by convection or evaporation. The bigger the wind, the greater the heat release that occurs. Each increment of $4.6 \mathrm{~m} / \mathrm{s}$ for wind speeds above $10 \mathrm{~m} / \mathrm{s}$, will lower the temperature by $10^{\circ} \mathrm{C}$. Indeed, this cannot be used in the house environment since the wind speed of that size will lead to disruptions, such as fallen trees. The wind flow in the housing area is affected by the density factor of the building and vegetation. As the wind blows through open surfaces in urban areas, the surface roughness is determined by the building and its structure. The denser the urban environment, it will reduce the wind speed in the area due to its roughness is also increasing. However, this influence depends on the variation of urban physical formation and the building layouts. When the building is placed in a row, like a row house pattern, it will cause a windbag and a little wind flow. In general, if the group of buildings is placed symmetry then the potential use of natural cross ventilation is reduced. Vegetation affects airflow through filtering, reflection, and breaking. This effect will increase and decrease the wind speed. 


\subsection{Tropical Nusantara's house characters}

The area of Indonesia in the humid tropics has characteristics, among others: relatively high air temperature and temperature difference between day and night is relatively small, strong wind blow during the day, meanwhile in the morning and evening is relatively low, has air humidity which is relatively high with a range between $60 \%-95 \%$ and relatively high solar heat radiation greater than $900 \mathrm{~W} / \mathrm{m} 2$. This character mainly affects the shape of the house in the tropics with the presence of air circulation. In spaces that have openings or that are in the vicinity of buildings, in general, have a shade, this can be used to avoid in-building heating due to direct solar radiation that generally occurs two hours at midday. Given considerable solar radiation, the selection of materials for building material also needs to consider absorption and reflection factors to help reduce solar radiation that can heat the building.

Natural cooling is a basic and long-standing method used to achieve comfort in humid tropical temperatures. The main step in natural cooling of tropical buildings is the shade. If the eaves or shade protrudes along the half of the height of the window, it will overshadow the window completely. Window and wall types of lattice with vines can also be used as a shade on the facade of the building. Strategy using landscape elements is the use of plants that overshadow the Northern and Western walls for the building position on the Southern latitudes of the equator. The shade design on the East and West wall requires a different approach because the sun position at rising and sinking is below the horizon, the eves are not effective to prevent sunlight. Vegetation may be the most effective way of keeping the intensity of the morning and afternoon sun that hit the East and West walls and windows. However, this way should also consider the vegetation function as a wind direction which is one of the other cooling strategies. If vegetation is unusable, then a combination of light colors, reflective glass, and a shielding grille is an effective strategy.

Another Tropical Nusantara's house character is in its materials. Houses with materials that have large thermal mass, such as walls and stone floors serve as long-lasting heat sinks to create optimum cooling inside the room. However, the environment surrounding the building must be designed to minimize the solar radiation that hit the surface of the building and prevent any radiation and heat reflection in the area. Glass materials should be reduced in use on the roof, Western and Eastern walls where the heat of the sun is greatest. The afternoon sun has a negative impact on skylight and roof openings as well as increasing heat reception. Skylights and roof openings should not be used unless they have been insulated or received imagery North-facing vertical glass (windows and walls) with eaves or shading indicates fewer problems, but still affects the inside temperature of the building. The convection cooling model is one of the most commonly applied cooling models in the tropics besides the radiative and evaporative cooling models. The convection cooling model is a heat acceptance adjustment model that is made to keep the room temperature comfortably in the building by using the natural carriage. Natural tolerances used for natural cooling also allow for the exchange of air at the right time. Convection model is based on cold air principle that can push hot air. The open window will release hot air near the ceiling to exit the building. The cold air will then enter the building through an open ventilation and replace the hot air. A simple formula to get the best cooling value is the number of openings should be between $50 \%$ to $100 \%$ more than on the sides of the entrance. If the tread or area there is only a breeze with a speed less than $0.25 \mathrm{~m} / \mathrm{s}$, then natural convection can still be used floating avenging. The buoyancy of floating is the exchange of air and cooling in space through a considerable temperature difference between the inner and outer space. As the hot air rises, the opening at the top allows hot air to exit the building so the cold air enters through lower ventilation and replaces the hot air. The coldest air in the building in the tropics is generally the Southern side or the area affected by the imagery and has a water element. The entry of cold air through the best 
ventilation is on the South side. The greater the temperature difference in the lower and upper ventilation, the natural airflow gets faster and more heat dissipation occurs. There are two basic ways to enhance the wind load of convective cooling, i.e. by increasing the volume of hot air coming out and the volume of cold air coming into the building. Ventilation at the rooftop is one way to increase airflow and increase the cooling load. A better approach is to use solar radiation to induce a faster flow with the use of a solar chimney. The simple principle of the solar chimney is to enter the sunlight on the roof of the chimney that consists of space above the roof until the temperature reaches $50^{\circ} \mathrm{C}$. This hot air then rises quickly and out, allowing cold air to enter the house through ventilation on the lower wall. Research conducted by the author in 2007 shows the application of solar chimneys in the tropics capable of producing a maximum speed of $0.8 \mathrm{~m} / \mathrm{s}$ in the room.

The main things to consider in designing a climate-friendly tropical building include: minimizing heat recovery during the day and optimizing heat release at night; minimize heat in the room; choose a site that complies with microclimate criteria; optimizing building materials especially in the slowdown of heat propagation time; control of solar radiation; air circulation arrangement. Some building designs for humid tropical climatic conditions are removing most of the heat energy generated by environmental temperatures and the intensity of solar radiation. The interaction of buildings against solar radiation is the largest source of heat in the room in buildings. The natural way that can be done to cool the building is to minimize solar radiation, determine the right building orientation, provide adequate distance to the surrounding buildings, and use shade elements to control the entry of solar radiation into the building. If the ambient temperature is higher than the temperature in the room, then the heat will enter the building through ventilation openings. This needs to be reduced by optimizing the elements of the building in achieving the resilience of the occupants.

\section{Tropical Nusantara's house strategy for contemporary design}

Tropical Nusantara's house design is not only to design the living space but also to make space a place that gives comfort and happiness. To achieve this, it is necessary to have elements creation and building systems that will be applied to the house. Spatial arrangements include roofs, walls, windows, and garden.

\subsection{Tropical Nusantara's roof}

Vertical sunlight during the hottest hours of the day causes the roof to bear the enormous heat intensity, so that the tropical roof design should be able to optimize natural cooling in the house by several elements, such as shading, reflection, and insulation that variations can be in roof shape, roof colors, and roof materials. The large roof area is recommended to be able to shade around the building and protect the outer walls against rain. The shape of the sloping roof is recommended for the anticipation of tropical rain, strong winds, hurricanes, and even the whirlwind. The roof with aluminum material has a weakness because of its nature as a good conductor of heat and easily corrosive due to its contact with sulfur dioxide substances contained in the atmosphere. Flat roofs made of concrete often cause problems in mainly dealing with depreciation and expansion processes. Construction of the roof with double sheets between the primary and secondary layers that allow airflow to occur in buildings is important. This construction prevents the sunlight glare during irradiation and direct heating on the outer layers. The choice of a bright roof color can reflect unwanted heat. The reflected power of a surface is measured by the amount of energy that can be absorbed and emitted. There are three main principles of Tropical Nusantara's roof design that includes shading, reflection, and insulation. Sunlight exposure 
to the roof can control the incoming solar radiation into the chamber, reducing cooling loads as well as increasing the thermal comfort and natural lighting qualities in the room. Appropriate external shading devices can control the amount of solar radiation admitted into the room, which could largely reduce cooling loads and improve indoor thermal comfort and daylighting quality. Bouchlaghem presented a computer model, which simulate the thermal performance of the building taking into account design variables related to the building envelope and optimize window-shading devices with optimization programs [1]. Corrado evaluates the influence of the geometry of window-shading device system on the thermal performance [2]. The reflection of sunlight on the roof can occur when the outer surface of the building is coated with colors that reflect solar radiation so that the heat transmission into the building should be reduced as much as possible. An alternative method can be done by providing vegetation shade in the form of trees or plants. As a result of the evaporation process performed by leaf surfaces in plants, the surface temperature of the building will be lower than the air temperature during the day and the temperature at night can be lower than the ambient air temperature. The heat insulation on the roof is the surface color and size of the insulation thickness. In general, insulation with a thickness of $5 \mathrm{~cm}$ is not enough to be used on the roof is red and blue colors. Insulation thickness at least has a minimum size of $8 \mathrm{~cm}$ for medium colors. Buildings with good roof insulation have temperatures ranging from $1{ }^{\circ} \mathrm{C}$ to $1.5^{\circ} \mathrm{C}$ lower than other buildings. The use of a green roof can serve as a good insulation system through the mechanism of constant heat changes on the building roof. Parameters of green roof design as an insulation related to the thickness of the soil layer, soil density, and soil moisture content. The tropical roof has a characteristic: brewing, attic or roof volume is large, allowing the disposal of hot air under the roof with openings or pores of the roof. Aspects of Nusantara include the characteristics of shelter is not only for protection and more on the exterior appearance than the interior.

\subsection{Tropical Nusantara's wall}

Tropical Nusantara's wall is required to create the average temperature and humidity conditions in the building to remain stable and unaffected by changes that occur in conditions outside the building. Building materials, such as concrete, bricks, and stones have a high thermal period. This is in contrast to materials with low thermal mass, such as wood and steel. This material has the ability to absorb heat quickly and cool it back quickly. Material selection becomes an ideal problem-solving in the cooling or warming of a building depending on its local climatic conditions. In humid tropical climate conditions, there are four factors in the creation of tropical wall design, namely: sun exposure to the wall, reflection of the sunlight, thermal material, and thermal insulation. The sun exposure to the wall is greater than the sun protection on windows. Room with walls without sun protection will be hotter by $2^{\circ} \mathrm{C}$ compared to building with sun protection. The most important treatment that can be done on buildings in the tropics is to protect most of the walls in East and West orientations that get more sunlight. Liping study on facade designs to improve indoor thermal comfort for naturally ventilated buildings, especially for hothumid climate [3]. In addition, it is noticed that there are very few guidelines for facade designers of naturally ventilated buildings or for occupants with the operation of an individual control over their thermal environment for the tropical climate. In tropical buildings located in the South of the equator, then the protection of the Northern wall is also important and vice versa. The reflection of sunlight on the wall can be done as a second creation by using surfaces that have high reflectivity. The use of white color on walls to reflect light is the best choice. Based on traditional building examples, lightweight thermal materials, such as wood, are suitable for hot-humid climates, especially at night 
when the temperature in the room decreases drastically and wind speed decreases. High thermal buildings perform the cooling process more slowly at night causing discomfort at bedtime. Over a 24-hour period of high thermal buildings can cause uncomfortable times to increase. However, during the day, the buildings have more advantages. So, if there is a natural carriage at night in the high thermal building, then it will be more comfortable compared with low thermal buildings. The choice of the thermal mass of the walls in the tropics for optimal natural cooling has two alternatives. The first alternative is using large thermal materials with natural nigh time strategies. While the second alternative is a mild thermal wall but requires a natural airtime strategy in the daytime. Based on the above explanation, it is obtained some sense that the building wall is influenced by three mechanisms of heat transfer, namely conduction, convection, and radiation. The acquisition of solar radiation on the external surface wall of the building will be converted into heat into the building by absorption and heat transfer by conduction. At the same time, heat transfer also occurs through the air outside the building to the outer wall surfaces and inner wall surfaces into the air inside the building. This leads to the acquisition of heat from the outside of very large building walls by conduction through the building walls as well as air leakage when the area within the building has a lower temperature. The first attempt in the heat prevention into the building is the protection of the building wall using the shade. The second attempt is the selection of the material period. Meanwhile, the last attempt to reduce the flow of heat from the outside into the building is using insulation materials. The thermal performance of building envelope is determined by the thermal properties of the materials used in its construction characterized by its ability to absorb or emit solar heat in addition to the overall U-value of the corresponding component including insulation. The placement of insulation material within the building component can affect its performance under transient heat flow. The best performance can be achieved by placing the insulating material close to the point of entry of heat flow. However, for practicality, it is common to use insulation to the inside or between wall cavities [4]. Selection of shade type, material term, and suitable insulation will affect the shape of the wall and its thickness to get a more efficient cooling system. The shape and thickness of the wall will increase the investment cost, but it will reduce the cost of energy use. Structures attached to the building have proved very helpful in reducing direct heat gain in the buildings [5]. So, at the optimum point of the wall design strategy will contribute enormously to the overall cost savings. Tropical Nusantara's wall is characterized by breathing, more optimizing the existence of windows on the North/Southside, openings forming angles with wind direction, the variation of openings position at the top - middle - bottom, and the use of lightweight materials.

\subsection{Tropical Nusantara's window}

In traditional buildings, the designer places window openings at certain points to create airflow. So far, window openings can reduce heat and humidity. But on the other side, the existence of these windows can increase the temperature in the room due to the entry of solar heat. The window opening on the East and West sides is the main part that needs to be protected, as the heat of the sun is higher in those orientations. Planting trees around the building are one way of shading by the trees to control the temperature by storing and releasing sunlight. Trees can also act as a cover against dust or something that easily enters through a window opening. There are two factors that are used to provide window openings in the tropical building principles, there is the sun and the size of openings. Some of the previous research results show a temperature rise of $2.5^{\circ} \mathrm{C}$ in the bedroom area with a shade less window opening facing North at the peak heat. The same is also happening in window opening without sun protection facing East with the excess heat above $3^{\circ} \mathrm{C}$. So, it 
is advisable that all windows have a type of protection system, such as shading devices and eaves. The main factors that influence the inclusion of daytime heat in the building are the size, shape, position of window openings, and the depth of the room. It is also influenced by factors that include the process of heat flow on the glass as well as outside aspects of buildings, such as the location of buildings and vegetation. Generally, the comparison between the height and width of the same window will allow the light to enter evenly into the room. Cross-ventilation is important in humid tropical climate after protection system against the heat of the sun. Natural ventilation or natural cooling system in the building is efficient in a humid tropical climate but still needs to pay attention to ambient air temperature. The main purpose of window openings design is to prevent excess heat in the room, so the temperature in the room is lower than outside of the room. Several studies have shown that indoor temperature comparisons show a difference of $1.5{ }^{\circ} \mathrm{C}$ between buildings with cross-ventilation systems compared to non-cross-ventilation systems [6]. Prianto examined various types of louver to improve the comfort level by use of increased air velocity [7]. Louver window at the ceiling height and floor level with an angle of 45 degrees achieve a comfortable condition under activities of 1 and 1.25 meeting. The modification of the ceiling height at the balcony and the enlargement of the opening dimension on the façade have no significant effect on the indoor comfort level. Actually, for heavyweight construction types smaller opening areas provided better performance also for free-running operation. Based on these simulations, a $50 \%$ value was set as a maximum window opening area in terms of wall area for dual mode operation [8]. Dividing the window area into several surfaces, preferably on opposing walls, is often considered favorable since it gives a more even and pleasant impression [9]. Ultimately, the window opening planning strategy includes the position of the windows on the East and West sides should be protected with a fairly wide shading device. The use of outside shading devices is better when compared to the use of deep indoor shading. This is because the use of deep indoor shading will still trap the heat inside the window.

\subsection{Tropical Nusantara's garden}

The main function of the tropical garden is as a natural cooling area outside the building so the incoming air becomes colder. Planting trees around the building are one way of controlling the temperature in repositories and keeping the sunlight out as well [10]. The tropical garden design consists of artificial and natural elements that both can control the microclimate and can reduce the amount of heat load into the building [11]. There are three factors that influence the principle of tropical garden, namely shading, reflection, and insulation or heat absorption. Shading creation using trees and garden-made elements, such as pergolas, is a very effective method of lowering the air temperature outside the building and protecting buildings from the heat of the sun. The best position in placing the plants is determined by reviewing the elements of the walls, roofs, and windows that get the most sunlight in the hottest months. The plotting of plants on the East and West sides receives more sunlight (about 50\%) than the orientation to the North and South. Trees should be planted in a predetermined position based on the current position of the hour and the time of solar radiation. Use of vines and creeping can be done with great advantage in its ability to protect most building facades in a short time. The plants are also useful in situations where there is a limited land. The reflection of sunlight is determined by the placing of artificial garden elements around the house. Surface soils that use paving can absorb and radiate large heat. The design of the garden aims to minimize the surface of a potential to collect heat. The use of paving that needs to be combined with grass that forms the pattern and shaded by shading trees or vines. The color of the paving surface also has an important 
role in absorbing and reflecting heat. Bright colors with rough surfaces can be used to the reduced glare of sunlight. Additional structures, whether or not neatly arranged, can be used to reduce absorption and reflection on the floor surfaces (roofs, walkways, and road for vehicles). Absorption and heat insulation on the floor of the building affected the ability of the earth surface to store heat. At a depth of 15 meters, the earth has a fixed temperature during the day. If some buildings are immersed in the ground, the building will release heat to the earth during the day and vice versa at night. Best absorption and heat insulation are plants. Solar thermal radiation is absorbed by the leaves through photosynthesis and heat evaporation. So, it not only becomes insulation but also converts solar energy into another useful energy. Tropical garden creations are creating an area that is overshadowed by construction elements or shade plants and the use of low absorptive materials and highly reflective, can minimize excessive solar radiation. The color of the plant and the surface on the floor around the building is also an important thing related to its ability to absorb solar radiation with different amounts. Colour, surface reflection, the number of protected areas of the building, soil density, and change in infrared radiation, they will affect the evaporation process on the surface. This evaporation process is an influential aspect in reducing average air humidity as surface temperature increases. Tropical gardens are characterized by the selection of shade plants; plants position on the East-West sides; priority of heat absorber plants, $\mathrm{CO} 2$, moisture; O2 producing plants; floor elevation for moisture anticipation and moisture absorbent floor materials. The aspect of Nusantara lies in the selection of productive plants that the fruits or leaves can be utilized by the surrounding communities. Plants should also be appropriate to the conditions and needs of the site. The main creation of Tropical Nusantara's house elements is to give influence to the environment outside and inside the building. The decrease in temperature outside the building will contribute to creating a better macro climate for the region. While the influence on the building will obviously increase comfort for the occupants. The integration of tropical house design can be explained in the following figure:

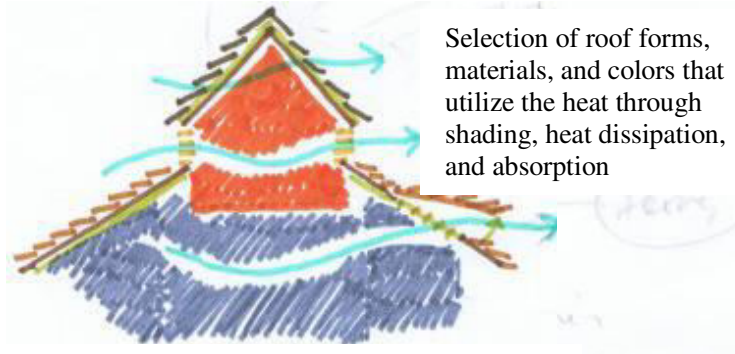

Selection of slope level, hollow type, materials, and colors of the wall that is utilizing solar heat through self-shaded, including wind and heat circulation

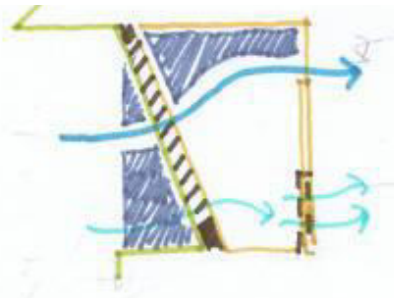

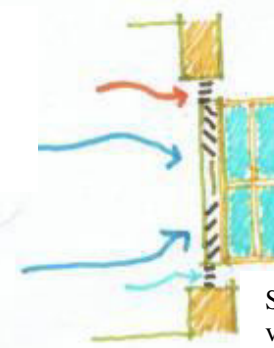

vegetation type and color as well as artificial elements, such as water and the type/color of surface shading materials, wind circulation, heat, and humidity

Fig. 2. The integration of Tropical Nusantara's House Elements. 


\section{Tropical Nusantara's contemporary house as cultural knowledge of living}

At the end of this paper, some notations that can be made include the living space, comfortable living, and cultural knowledge of living on Tropical Nusantara's house. Indeed, this is related to the initial problems we are facing not only about the identity or alternatives of present style but also more than that, as an effort to maintain the living comfort and sustainability of cultural knowledge of living as the essence of the house existence. As for the first series, we also offer stages to be done when we choose Tropical Nusantara's house as an alternative to the present style, as follows:

\section{First, Identify the Building Climate}

Recognize the tropical climate with steps: setting the direction and angle of solar radiation coming through overhangs and sun shading devices design to control the heat of the sun; setting the orientation of the building by minimizing the confrontation to the East and West, thus reducing direct sunlight and reducing heat and ultraviolet rays; the design of overhangs or external protectors to reduce heat and protect buildings from rain; arrangement of orientation, position, and building openings to obtain natural air; ventilation; integration of the internal open space (atrium, void) with roof or floor ventilations; optimization of passive cooling systems from landscaping and infrastructures; design of protection system of sun and rain in open areas and meeting rooms; use of external protector, such as roofed corridors that connect between buildings; use of insulation in the form of natural materials on the building skin; use of ponds, water elements, and vegetation to regulate air cooling through space; use of an effective elongated shape to regulate cross-ventilation; use of material colour as a sun reflector to reduce heat; use of energy-saving and maintenance-efficient materials.

\section{Second, Develop the Occupants Comfort}

The occupant's comfort in the tropics are highly dependent on the activities type that is tied to lifestyles, comfortable standards, and social culture. Aspects of Nusantara's occupants are the life together, simplicity, and togetherness in settlement environment area. The comfort of living in the house is in each room inside the house. The bedroom as a private room with larger climate protection that supports the tradition of resting of its inhabitants. Family room as a shared space as well as house climate area and the transfer of family knowledge. Service room area as a protective room from the climate as well as isolation. Terrace and garden as a social space that contributes to environmental comfort and social interaction. Meanwhile, the building element as the shaper of the space must also give more than just comfort. For example, roof and wall elements. Tropical Nusantara's roof is not only protecting from rain and heat, but it is also easy to care, giving shade, contributing in upper lighting, giving calmness, giving memories, and giving a knowledge about noble values of tropical nuance. Tropical Nusantara's wall, aside from protecting from the wind and noise, as well as a means of communication between neighbors with a formation that is not too massive and there many other examples.

\section{Third, Tropical Nusantara's Sense Habitation}

Efforts to achieve the satisfaction of occupancy can be achieved when looking at the tropical climate not as a constraint of air temperature and high humidity, but as a potential abundance of solar radiation (not heat radiation) and wind potential. Moreover, the aspect of Nusantara as a contemporary lifestyle that refers to the positive values of house tradition of the past is seen as the solution of future problems, where the meaning of the house as a place to live together, give to each other, to form the next generation independence that is adaptive and dynamic. Tropical Nusantara's nuance habitation has the main character that 
appears during the elongated to West-East orientation buildings, allowing evenly air circulation between buildings and utilizing the remaining space for the courtyard or garden between buildings. This gives an effect not only can utilize the air circulation evenly and mutual shade between buildings, but also provide space together and interaction between occupants of the settlement environment area. Other Tropical Nusantara's characters are in the aspect of space with unshaded features but not closed, have contact with the external environment, use of sunlight, use of color psychology - cool shade and slim modular. Nusantara's aspect appears in the spirit to produce space instead of consuming space, flexible and dynamic space, the existence of a common space, the importance of transition space. It is time, we are together in a variety of living spaces, embody the Tropical Nusantara's house that is responsive to climate, comfortable, and pleasant to live in the present and the future (figure 3).

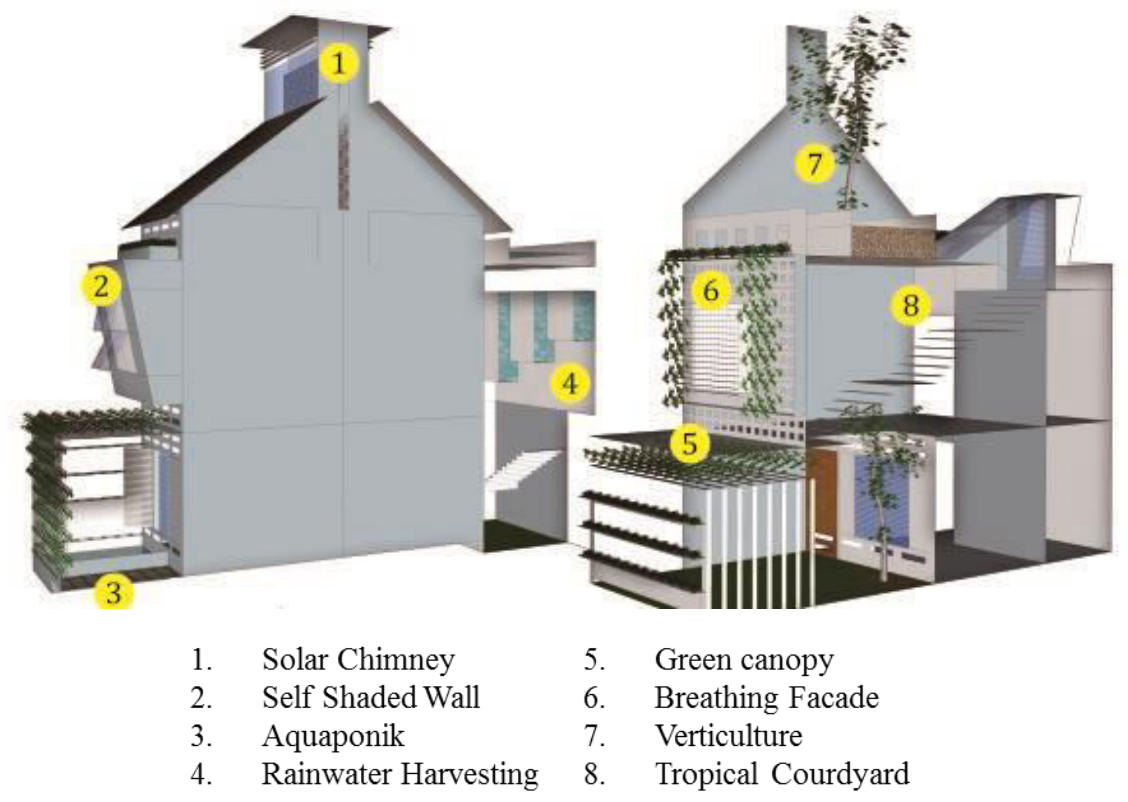

Fig. 3. The Idea of Tropical Nusantara's Contemporary House Model

\section{References}

1. N. Bouchlaghem, Optimizing the design of building envelopes for thermal performance, Automation in Construction, 10 (2000)

2. V. Corrado, V. Serra, A. Vosilla, Performance analysis of external shading devices, Proceedings of PLEA, Netherlands (2004)

3. W. Liping, W.N. Hien, The impacts of ventilation strategies and facade on an indoor thermal environment for naturally ventilated residential buildings in Singapore, Building and Environment (2007)

4. Al-Homoud, Performance characteristics and practical applications of common building thermal insulation materials, Building and Environment 40 (2005)

5. M.M. Bajwa, The role of integrated landscape design in energy conservation in detached dwellings in the Arabian Gulf region, Renewable Energy 6 (1995) 
6. F. Garde, Implementation and experimental survey of passive design specifications used in new low-cost housing under tropical climates, Energy and Buildings 36 (2004)

7. P. Prianto, Depecker, Optimization of architectural design elements in the tropical humid region with thermal comfort approach, Energy and Buildings 35 (2003)

8. T. Rosangela, Dual mode cooling house in the warm humid tropics, Solar Energy 73 (2002)

9. B.H. Helena, Energy efficient window systems: effects on energy use and daylight in buildings, unpublished doctoral thesis, Division of Energy and Building Design. Lund University, Lund Institute of Technology, Lund, (2001)

10. P. Gut, A. Dieter, Climate-responsive building appropriate building construction in tropical and subtropical regions, SKAT (1993)

11. J.H. Parker, Uses of landscaping for energy conservation, Department of Physical Sciences, Florida International University, Miami, Florida (1981). 